\title{
Impaired skeletal muscle mitochondrial bioenergetics and physical performance in chronic kidney disease
}

\author{
Bryan Kestenbaum, ${ }^{1,2}$ Jorge Gamboa, ${ }^{3}$ Sophia Liu, ${ }^{4}$ Amir S. Ali, ${ }^{4}$ Eric Shankland, ${ }^{4}$ Thomas Jue, ${ }^{5}$ \\ Cecilia Giulivi, ${ }^{6}$ Lucas R. Smith, ${ }^{7}$ Jonathan Himmelfarb, ${ }^{1,2}$ Ian H. de Boer, ${ }^{1,2,8}$ Kevin Conley, ${ }^{4}$ \\ and Baback Roshanravan ${ }^{9}$ \\ 'Division of Nephrology, Department of Medicine, and ${ }^{2}$ Kidney Research Institute, University of Washington, Seattle, \\ Washington, USA. ${ }^{3}$ Department of Medicine, Vanderbilt University Medical Center, Nashville, Tennessee, USA. \\ ${ }^{4}$ Department of Radiology, University of Washington, Seattle, Washington, USA. ${ }^{5}$ Department of Biochemistry and \\ Molecular Medicine, School of Medicine, ${ }^{6}$ Department of Molecular Biosciences, School of Veterinary Medicine, and \\ ${ }^{7}$ Department of Physical Medicine and Rehabilitation, School of Medicine, UCD, Davis, California, USA. ${ }^{8}$ Puget Sound \\ Veterans Administration Healthcare System, Seattle, Washington, USA. 'Division of Nephrology, Department of Medicine, \\ School of Medicine, UCD, Sacramento, California, USA.
}

The maintenance of functional independence is the top priority of patients with chronic kidney disease (CKD). Defects in mitochondrial energetics may compromise physical performance and independence. We investigated associations of the presence and severity of kidney disease with in vivo muscle energetics and the association of muscle energetics with physical performance. We performed measures of in vivo leg and hand muscle mitochondrial capacity (ATPmax) and resting ATP turnover (ATPflux) using ${ }^{31}$ phosphorus magnetic resonance spectroscopy and oxygen uptake ( $\mathrm{O}_{2}$ uptake) by optical spectroscopy in 77 people ( 53 participants with CKD and 24 controls). We measured physical performance using the 6-minute walk test. Participants with CKD had a median estimated glomerular filtration rate (eGFR) of $33 \mathrm{ml} / \mathrm{min}$ per $1.73 \mathrm{~m}^{2}$. Participants with CKD had a $-0.19 \mathrm{mM} / \mathrm{s}$ lower leg ATPmax compared with controls but no difference in hand ATPmax. Resting $\mathrm{O}_{2}$ uptake was higher in CKD compared with controls, despite no difference in ATPflux. ATPmax correlated with eGFR and serum bicarbonate among participants with GFR $<60$. ATPmax of the hand and leg correlated with 6-minute walking distance. The presence and severity of CKD associate with muscle mitochondrial capacity. Dysfunction of muscle mitochondrial energetics may contribute to reduced physical performance in CKD.

Authorship note: $J \mathrm{G}$ and SL contributed equally to this work.

Conflict of interest: The authors have declared that no conflict of interest exists.

Copyright: (c) 2020, American Society for Clinical Investigation.

Submitted: September 6, 2019 Accepted: December 10, 2019 Published: January 30, 2020.

Reference information: /CI Insight. 2020;5(2):e133289.

https://doi.org/10.1172/jci. insight.133289.

\section{Introduction}

The maintenance of functional independence is the top health priority reported by patients living with chronic kidney disease (CKD) (1). Skeletal muscle impairment, which manifests as muscle loss or weakness (sarcopenia), is the final common pathway of multiple adverse metabolic processes that arise from kidney disease, including malnutrition-inflammation and oxidative stress. Greater insights into the mechanisms underlying skeletal muscle impairment in $\mathrm{CKD}$ are needed to motivate new therapeutic directions for preserving muscle function critical to independent living.

Experimental evidence suggests that impaired mitochondrial metabolism is a central mechanism linking kidney dysfunction with sarcopenia. First, animal studies demonstrate that kidney disease promotes deficits in mitochondrial electron transport and pyruvate dehydrogenase activity, which manifest as reduced endurance before the onset of muscle atrophy or weakness (2). Second, analyses of the plasma metabolome and interrogation of skeletal muscle tissue in persons with CKD reveal abnormalities in specific enzymes and metabolic pathways related to mitochondrial energy generation $(3,4)$. Third, lipidomic studies show that reduced kidney function is associated with disruption of mitochondrial fatty acid oxidation (5). Missing from these observations is direct in vivo evidence of altered muscle mitochondrial bioenergetics in patients with $\mathrm{CKD}$.

We applied dynamic procedures to quantify skeletal muscle mitochondrial energetics in humans using ${ }^{31}$ phosphorus magnetic resonance spectroscopy $\left({ }^{31} \mathrm{P} \mathrm{MRS}\right)$ and optical spectroscopy. Using these proce- 
dures, we compared key muscle mitochondrial characteristics: oxidative phosphorylation capacity (ATPmax) and oxygen uptake between people with and without CKD. We tested the hypothesis that the presence and severity of kidney disease is associated with worse impairment of in vivo muscle mitochondrial oxidative capacity. We then tested whether in vivo muscle mitochondrial oxidative capacity is associated with measured physical performance, determined by 6-minute walking distance.

\section{Results}

Participant characteristics. In total, 77 participants (53 participants with moderate to advanced CKD and 24 without $\mathrm{CKD}$ ) completed hand experiments and 59 participants ( 40 with CKD and 19 without CKD) completed leg experiments for ATPmax (Supplemental Figure 1; supplemental material available online with this article; https://doi.org/10.1172/jci.insight.133289DS1). The mean age of the study participants was $63 \pm 13$ years, and 38 (49\%) were female (Table 1). Median of estimated glomerular filtration rate (eGFR) among participants with CKD was 33 [interquartile range, 23, 49] ml/min/1.73 $\mathrm{m}^{2}$ compared with 102 [interquartile range 81, 108] $\mathrm{ml} / \mathrm{min} / 1.73 \mathrm{~m}^{2}$ among control individuals. Participants with $\mathrm{CKD}$ tended to be older, were more likely to be female, and had lower hemoglobin $(\mathrm{Hb})$ concentrations and higher $\mathrm{C}$-reactive protein concentrations compared with control individuals. The prevalence of diabetes was $36 \%$ in the CKD group and $33 \%$ in the control group.

CKD and muscle oxidative capacity. Forty participants with CKD and nineteen controls had measures of leg muscle oxidative capacity (ATPmax). Participants with CKD had a mean age of $62 \pm 14$ years, with a $55 \%$ prevalence of females, compared with $60 \pm 8$ years $(P$ for difference $=0.6)$ with a $32 \%$ prevalence of females $(P$ for difference $=0.1)$ in controls (Supplemental Table 1$)$. Participants with $C K D$ had a mean leg muscle ATPmax of $0.60 \pm 0.16 \mathrm{mM} / \mathrm{s}$ compared with $0.80 \pm 0.18 \mathrm{mM} / \mathrm{s}$ in control subjects. Participants with CKD had a mean hand muscle ATPmax of $0.88 \pm 0.29 \mathrm{mM} / \mathrm{s}$ compared with $0.94 \pm 0.27 \mathrm{mM} / \mathrm{s}$ in control subjects. After adjustment for age, sex, BMI, and history of diabetes, CKD was associated with a $-0.19 \mathrm{mM} / \mathrm{s}$, or $1 \mathrm{SD}$, lower leg muscle ATPmax (95\% CI 0.27-0.1 lower; $P<0.001)$ (Figure 1 ). No significant difference was noted in the hand muscle ATPmax of participants with CKD compared with controls. History of diabetes was also associated with a lower ATPmax after adjustment $(0.118 \mathrm{mM} / \mathrm{s}$, or $0.6 \mathrm{SD}$ lower ATPmax; 95\% CI 0.198-0.04 lower; $P=0.004$ ). The magnitude of association between CKD status and leg muscle ATPmax was numerically smaller among participants who had diabetes $(0.117 \mathrm{mM} / \mathrm{s}$ lower ATPmax; 95\% CI 0.023-0.21 lower) compared with those who did not have diabetes $(0.213 \mathrm{mM} / \mathrm{s}$ lower ATPmax; 95\% CI 0.097-0.328 lower; $P$ for interaction $=0.270$ ).

In subgroup analysis in which participants younger than 65 years $(n=36)$ were compared with participants aged 65 years and older $(n=23)$, CKD was persistently associated with lower leg ATPmax compared with controls, even after adjusting for sex, BMI, and diabetes status. Among participants younger than 65 years, CKD was associated with an estimated $0.178 \mathrm{mM} / \mathrm{s}(0.95 \mathrm{SD})$ lower leg ATPmax (95\% CI 0.06-0.29 lower; $P=0.003)$ after adjustment compared with $0.170 \mathrm{mM} / \mathrm{s}(0.9 \mathrm{SD})$ lower leg ATPmax (95\% CI 0.06 0.28 lower; $P=0.005$ ) among those aged 65 years and older.

When analyzed as a continuous variable, lower GFR was associated with lower ATPmax in the tibialis anterior leg muscle but not in the interosseous hand muscle (Figure 2). Each $10 \mathrm{ml} / \mathrm{min} / 1.73$ $\mathrm{m}^{2}$ lower eGFR was associated with a $0.028 \mathrm{mM} / \mathrm{s}(0.15 \mathrm{SD})$ lower leg muscle ATPmax after adjustment (95\% CI 0.015-0.04 lower; $P<0.001)$. In sensitivity analysis further adjusting for muscle size and muscle strength, lower eGFR remained associated with lower leg muscle ATPmax $(0.027 \mathrm{mM} / \mathrm{s}$ [0.14 SD] lower; 95\% CI 0.012-0.041 lower; $P=0.001$ ). When restricting analyses to participants with an eGFR $<60 \mathrm{ml} / \mathrm{min} / 1.73 \mathrm{~m}^{2}$, each $10 \mathrm{ml} / \mathrm{min} / 1.73 \mathrm{~m}^{2}$ lower eGFR was associated with a 0.039 $\mathrm{mM} / \mathrm{s}(0.21 \mathrm{SD})$ lower (95\% CI 0.003-0.074 lower; $P=0.03)$ leg muscle ATPmax after adjustment. Associations with whole leg muscle oxidative capacity, expressed as ATPmax*CSA, yielded similar results (Supplemental Table 2).

CKD and resting muscle ATPflux and oxygen uptake. Resting ATP turnover (ATPflux), which was measured only in the hand muscle, was performed on 70 participants (21 controls and 49 participants with CKD). Participants with CKD had a mean age of $63 \pm 15$ years, with a $59 \%$ prevalence of females, compared with $58 \pm 10$ years $(P$ for difference $=0.33$ ), with a $56 \%$ prevalence of females in controls (Supplemental Table 3 ).

Resting ATPflux was not meaningfully or significantly different between persons with and without CKD (Figure 3). Resting hand muscle oxygen uptake was performed on 49 participants ( 9 controls and 40 participants with CKD). Hand muscle oxygen uptake was $0.49 \mu \mathrm{M} / \mathrm{s}(0.56 \mathrm{SD})$ higher $(95 \% \mathrm{CI} 0.08-0.9 ; P=0.02)$ in CKD 
Table 1. Participant characteristics

\begin{tabular}{|c|c|c|}
\hline & CKD $(n=53)$ & Control $(n=24)$ \\
\hline Age in yr, mean (SD) & $64(14)$ & $60(9)$ \\
\hline Female, no. (\%) & $29(55)$ & $9(38)$ \\
\hline Black, no. (\%) & $7(13)$ & $3(13)$ \\
\hline Participants with diabetes, no. (\%) & $19(36)$ & $8(33)$ \\
\hline $\mathrm{BMl}$ in $\mathrm{kg} / \mathrm{m}^{2}$, mean (SD) & $30.1(7)$ & $28.3(6)$ \\
\hline Waist-to-hip ratio, mean (SD) & $0.96(0.09)$ & $0.94(0.08)$ \\
\hline Albumin in $\mathrm{g} / \mathrm{dL}$, mean (SD) & $3.9(0.3)$ & $4(0.4)$ \\
\hline Albuminuria in mg/g, median (IQR) & $244(27,678)$ & $5(1,10)$ \\
\hline Bicarbonate in mmol/L, mean (SD) & $22(3)$ & $23(2)$ \\
\hline Hemoglobin in g/dL, mean (SD) & $12.4(2)$ & $14.1(2)$ \\
\hline hsCRP, median (IQR) & $0.21(0.08,0.52)$ & $0.09(0.04,0.2)$ \\
\hline
\end{tabular}

${ }^{A} n=50$ CKD and $n=22$ control. ${ }^{B} n=37$ CKD and $n=19$ control. ${ }^{C} n=35$ CKD and $n=17$ control. eGFRcr-cysc, the estimated glomerular filtration rate from the combined creatinine and cystatin C equation; IQR, interquartile range; hsCRP, high sensitivity C-reactive protein.

patients compared with control individuals, and this difference was not materially altered by adjustment for age, sex, BMI, and ATPflux (adjusted difference 0.54 [0.62 SD] higher; 95\% CI 0.043-1.19 higher; $P=0.036$ ).

Correlates of muscle mitochondrial oxidative capacity. Leg muscle ATPmax was correlated with eGFR ( $r=$ $0.47, P=0.004)$ and serum bicarbonate $(r=0.39, P=0.02)$ among those with eGFR $<60$. In this subgroup, those with serum bicarbonate levels below the threshold recommended by Kidney Disease: Improving Global Outcomes (KDIGO) for bicarbonate replacement therapy $(<22 \mathrm{mmol} / \mathrm{L})$ had lower leg muscle ATPmax values compared with those with levels of $22 \mathrm{mmol} / \mathrm{L}$ and greater (Figure 4 ). There was no significant correlation between leg muscle ATPmax and $\log \mathrm{CRP}(r=-0.31, P=0.07)$ or log of urine albumin to creatinine ratio $(r=-0.33, P=0.05)$ in this subgroup. Correlations of leg muscle and hand muscle ATPmax with muscle characteristics and anthropometrics are listed in Supplemental Table 4. Hand muscle mitochondrial capacity was poorly correlated with plasma biomarkers $(r<0.15)$. There was also no significant or meaningful association of sex with muscle oxidative capacity ( $P=0.13$ for leg muscle and $P=0.8$ for hand muscle).

Muscle mitochondrial oxidative capacity and physical performance. Seventy-five participants completed a 6-minute walk test and had first dorsal interosseous (FDI) hand measurements of muscle ATPmax; of these participants, 57 completed tibialis anterior leg muscle ATPmax measurements in the study. The mean 6-minute walk distance was 1342 feet (95\% CI 1244-1438) among those with CKD compared with 1734 feet (95\% CI 1638-1830) among controls ( $P$ for difference $<0.0001$ ). Leg muscle ATPmax, and to a lesser extent, the FDI hand muscle ATPmax, were positively correlated with 6-minute walk performance (Figure 5). Each 1 $\mathrm{mM} / \mathrm{s}$ greater leg muscle ATPmax and hand muscle ATPmax was associated with 1086 feet greater $(95 \% \mathrm{CI}$ 642-1530 greater; $P<0.001)$ and 388 feet greater (95\% CI 111-664 greater; $P<0.001)$ 6-minute walk distance, respectively. These associations remained significant after adjustment (Table 2 and Table 3). There was no evidence for heterogeneity by CKD status for the association of leg muscle ATPmax with 6-minute walk performance $(P$ for interaction $=0.11)$. Similar associations were observed in sensitivity analyses between leg whole muscle ATPmax*CSA and 6-minute walk distance (Supplemental Table 5 and Supplemental Figure 2).

\section{Discussion}

Our findings demonstrate that the presence and severity of $\mathrm{CKD}$ is associated with decreased in vivo muscle mitochondrial oxidative capacity (ATPmax) in the tibialis anterior muscle, which is involved with mobility tasks. We did not observe associations of CKD with ATPmax in the FDI muscle of the hand; however, we found higher resting muscle oxygen consumption in hand muscles of patients with CKD without accompanying difference in resting ATPflux, consistent with prior investigations and studies of 

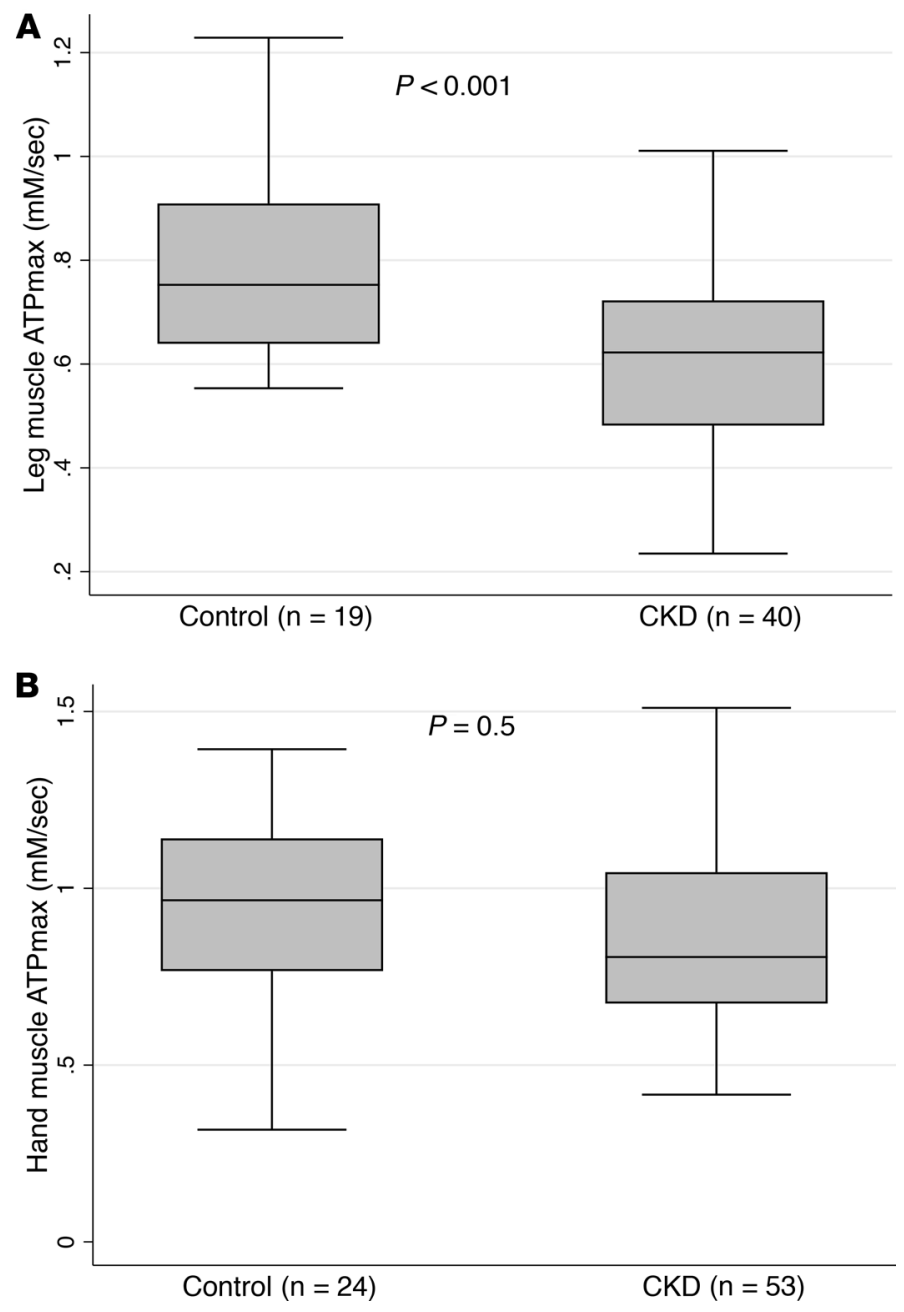

Figure 1. Chronic kidney disease is associated with lower leg muscle ATPmax but not hand muscle ATPmax compared with controls. Association of CKD with leg muscle mitochondrial capacity $(\mathbf{A})$ and hand muscle mitochondrial capacity (B). The shaded areas represent the interquartile range (25th-75th percentile) of the data, and middle horizontal lines represent the median value for each group. $P$ values were adjusted for age, sex, BMI, and diabetes using multivariable linear regression.

leg muscles of patients with kidney disease treated with chronic dialysis $(6,7)$, suggesting increased mitochondrial uncoupling. Measurements of mitochondrial energetics by ${ }^{31} \mathrm{P}$ MRS were directly linked with physical performance on a 6-minute walk test, indicating functional relevance and suggesting a link between mitochondrial function and skeletal muscle impairment in $\mathrm{CKD}$. Together, these findings build upon the body of existing evidence implicating impaired mitochondrial energetics as central mechanism linking $\mathrm{CKD}$ with decreased muscle performance.

The nature and extent of deficits in leg muscle mitochondrial metabolism observed in this study of CKD are consistent with those previously reported in maintenance dialysis $(7,8)$. This finding is important because dialysis per se is associated with transient alterations in phosphate homeostasis that may impair oxidative capacity and compound muscle metabolic injury, including intracellular inorganic phosphate depletion and reduced muscle phosphocreatine dynamics (9). Prior studies of patients treated with chronic hemodialysis demonstrated impaired muscle mitochondrial oxidative capacity in absence of any difference in muscle $\mathrm{pH}$ or deficits in oxygen delivery measured by near-infrared spectroscopy during prolonged exercise (8). Furthermore, improvement of the blood oxygen content with erythropoietin-stimulating agents does not improve peak oxygen uptake or muscle mitochondrial energetics by in vivo ${ }^{31} \mathrm{P}$ MRS among patients receiving dialysis $(10,11)$.

$\mathrm{CKD}$ is a catabolic and proinflammatory condition that may disrupt mitochondrial energy metabolism via several biochemical pathways (3). Uremic toxins, such as indoxyl sulfate, L-kynurenine, kynurenic acid, indole-3-acetic acid, and p-Cresol sulfate, adversely affect mitochondrial metabolism $(12,13)$ and insulin resistance (14). Exposure of isolated skeletal muscle mitochondria from the C57BL/6N mice to uremic toxins leads to deficits in the electron transport system characterized by decreased complex III and IV activity in addition to increased $\mathrm{H}_{2} \mathrm{O}_{2}$ production (13). Uremic toxins can also impair UDP-glucuronosyltransferases critical for the metabolism of drugs and toxins, the buildup of which can lead to impaired mitochondrial oxidative phosphorylation (15). CKD may also promote impairment in the switching from anaerobic to aerobic carbohydrate metabolism. Murine 5/6th nephrectomy models demonstrate inactivation of mitochondrial pyruvate dehydrogenase activity (2), the entry point into the Krebs cycle. Moreover, metabolic acidosis, the extent of which may not be reliably indicated by serum bicarbonate concentrations, has also has been purported to contribute to insulin resistance and protein degradation through glucocorticoid-dependent mechanisms (16) and augmentation of ATP-dependent proteolytic pathways (17).

We observed that, in the subgroup with eGFR $<60$, those with serum bicarbonate levels below the threshold recommended by the KDIGO guidelines for bicarbonate replacement therapy (18) had substantially lower leg muscle ATPmax values compared with those above the threshold. Current practice guidelines recommend that people with $\mathrm{CKD}$ and serum bicarbonate concentrations $<22 \mathrm{mmol} / 1$ should be treated with oral bicarbonate supplementation to maintain serum bicarbonate within the normal range. These guidelines have been informed by animal studies and clinical trials targeting metabolic acidosis in CKD that have demonstrated improvements in muscle mass, physical performance, and physical function $(19,20)$. In animal models of $\mathrm{CKD}$, metabolic acidosis suppresses the effects of the insulin receptor substrate (IRS)/phosphatidylinositol 3-kinase (PI3K)/AKT pathway and upregulates transcription of genes that code for the ubiquitin proteosome 

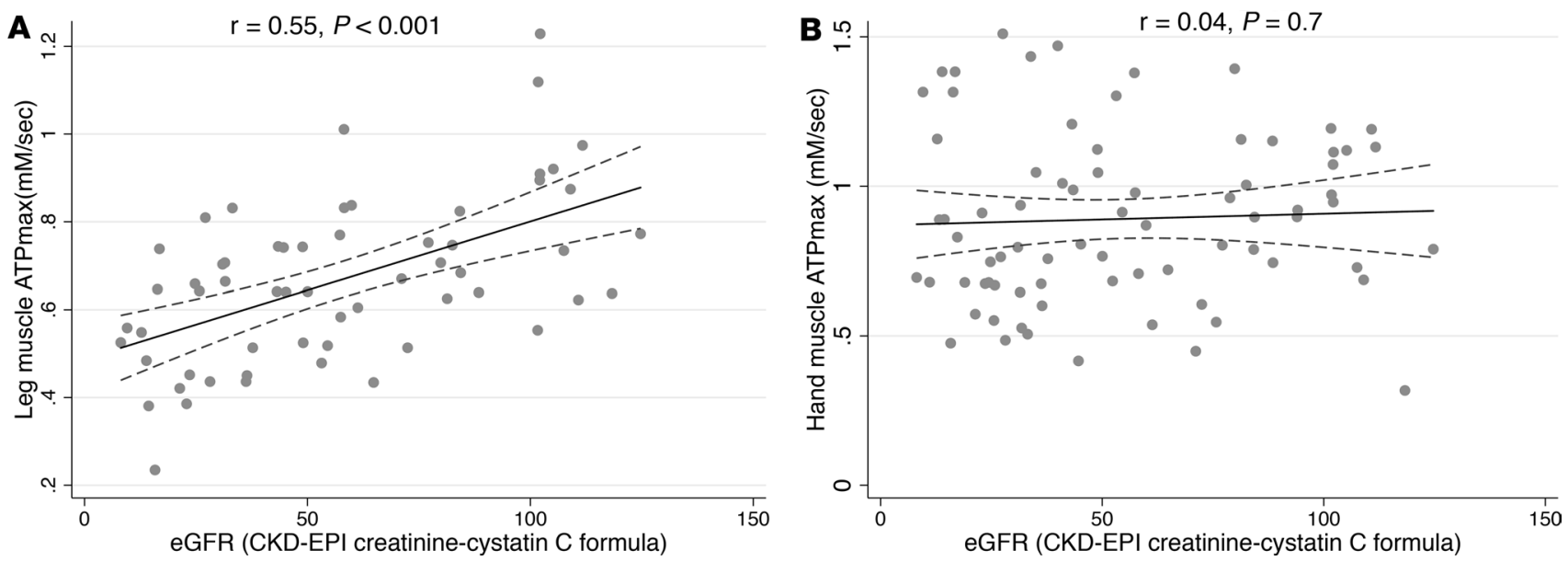

Figure 2. Kidney function is associated with in vivo leg muscle mitochondrial capacity but not hand muscle mitochondrial capacity. Association of eGFR with leg muscle (A) and hand muscle mitochondrial capacity (B). $P$ values were obtained using linear regression.

system, contributing to skeletal muscle proteolysis $(17,21,22)$. Inhibition of the PI3K/AKT pathway can also lead to decreased activation of the ATP citrate lyase, which impairs cardiolipin synthesis 42 and mitochondrial supercomplex formation, complex levels, and activity necessary for mitochondrial membrane integrity and electron transport chain function (23). Our findings motivates further investigation of pharmacologic and lifestyle interventions targeting metabolic acidosis to attempt to reverse impairments in muscle mitochondrial metabolism and muscle function in persons living with CKD.

Oxidative stress associated with CKD may also contribute to mitochondrial dysfunction through activation and insertion of uncoupling proteins into the mitochondrial membrane (24), leading to increased oxygen consumption without concomitant increase in ATPflux (uncoupling of oxidative phosphorylation), as has been observed in patients with nondiabetic CKD and in leg muscles of patients with end-stage kidney disease treated with dialysis (6). Our findings of impaired in vivo muscle mitochondrial oxidative capacity and possible pathologic dyscoupling of mitochondrial oxidative phosphorylation motivate investigation of mitochondrial targeted therapeutics and their effect on muscle mitochondrial energetics and muscle performance in patients living with CKD. One potential therapeutic is elamipretide, an aromatic-cationic, cell-permeable tetrapeptide mitochondrial targeted drug shown to improve mitochondrial efficiency, oxidative stress, capacity, and muscle endurance in preclinical models of aging and limb immobilization $(25,26)$.

Our findings are also consistent with metabolic studies of CKD that suggest aberrant mitochondrial function. The most prominent abnormalities observed in the fasting plasma metabolome among patients with CKD include elevation in fumarate and disruption in the ubiquinone biosynthesis pathway (3). Ubiquinone is vitally important for shuttling of electrons among electron transport chain complexes I, II, and III. Depletion of coenzyme Q, a product of ubiquinone biosynthesis, has been linked to increased electron leak with generation of superoxide from complex II (27), while elevated fumarate has been associated with depletion of glutathione and redox senescence (28). These findings further motivate investigations of in situ muscle mitochondrial energetics using high-resolution respirometry to examine dysfunction in complex I and II and assess the redox status of the ubiquinone pool in patients living with CKD versus controls. A natural extension of our investigation will be testing reversibility of mitochondrial energetic deficits in ongoing clinical trials of exercise in patients living with CKD (ClinicalTrials.gov NCT02923063) or coenzyme Q or nicotinamide riboside supplementation (ClinicalTrials.gov NCT03579693).

In contrast to the leg muscle, we did not observe differences in mitochondrial oxidative capacity in the hand muscle between persons with and without CKD. Similar findings have been reported using ${ }^{31} \mathrm{P}$ MRS in patients receiving maintenance hemodialysis (29). There are several potential explanations for the discrepant associations in hand and leg muscles. The first is a technical limitation of the MRS procedure in the much smaller dorsal interosseous muscle, which has a lower signal-to-noise ratio for ${ }^{31} \mathrm{P}$ MR spectra that may limit the ability to detect differences in phosphocreatine recovery with exercise. Second, the tibialis anterior leg muscle contains predominantly type I fibers, which have greater mitochondrial volume 
A

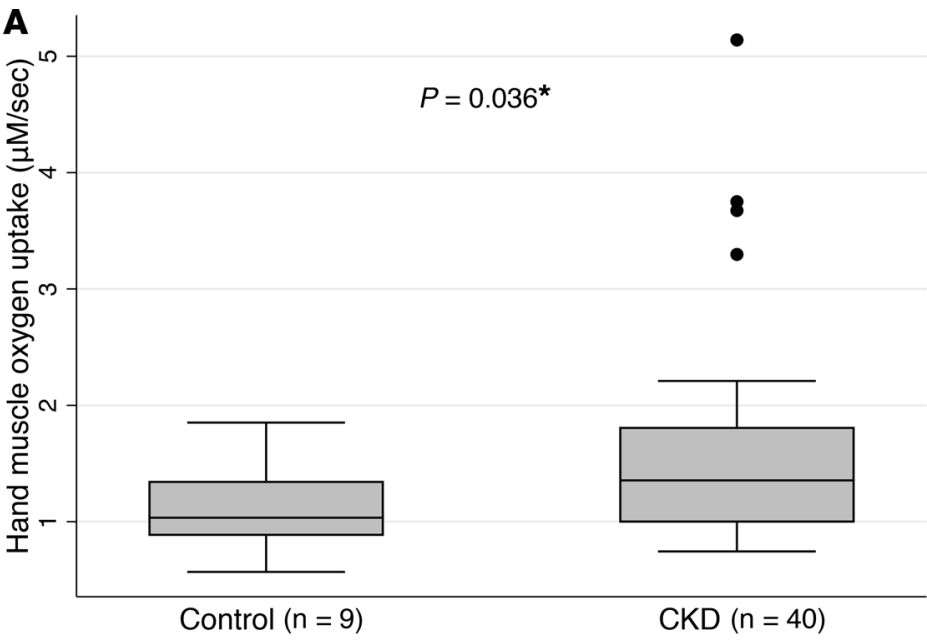

*Adjusted for age, sex, BMI, and resting ATPase

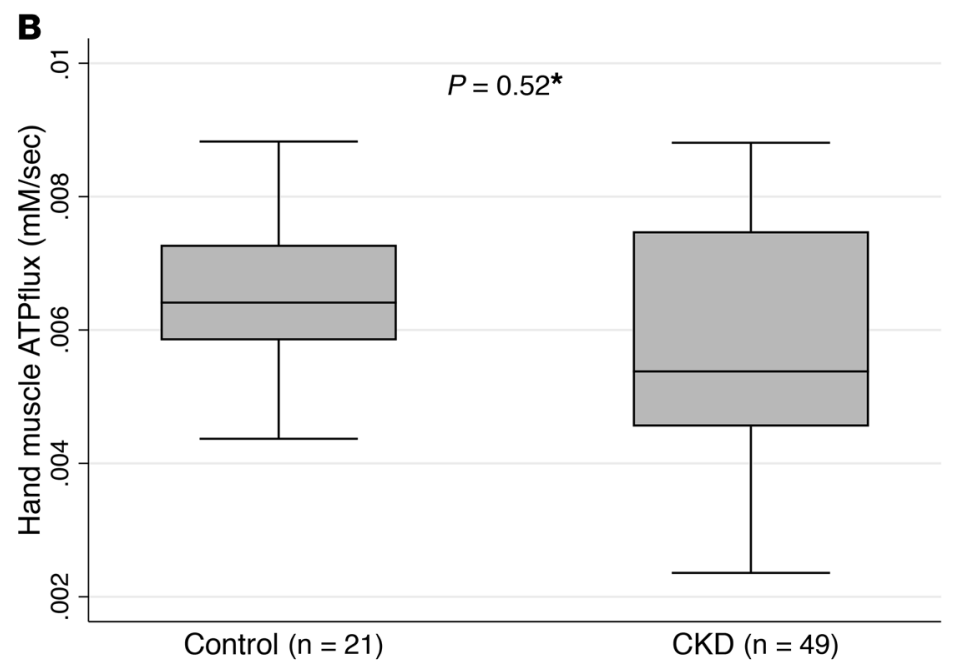

*Adjusted for age, sex, and BMI
Figure 3. Chronic kidney disease is associated with greater resting hand muscle oxygen uptake, with no differences in resting ATPflux compared with controls. Box plot of the association of CKD with resting hand muscle (A) $\mathrm{O}_{2}$ uptake and (B) ATPflux. The shaded areas represent the interquartile range (25th-75th percentile) of the data, and middle horizontal lines represent the median value for each group. Hand muscle $\mathrm{O}_{2}$ uptake was adjusted for age, sex, BMI, and ATPflux. $P$ values were obtained using multivariable linear regression.

and a greater dependency on oxidative metabolism (30). In contrast, the dorsal interosseous muscle consists mainly of type II fibers, which have a higher glycolytic capacity, with low-to-moderate oxidative capacity (31). These differences combined with potential differences in habitual use of these muscles in daily activities may explain the discrepancy in associations with kidney dysfunction. Although kidney disease patients demonstrated greater oxygen uptake under resting conditions where membrane potential is high, this does not necessarily translate into reduced ATPmax measured under high-flux conditions with lower membrane potential during exercise. Since membrane potential is a key driver of unregulated proton leak across the mitochondrial inner membrane (32) and proton leak is an important mechanism underlying reduced coupling (33) (stable ATPflux despite increased oxygen uptake), the coupling of ATP production to oxygen uptake is expected to increase as the flux through the electron transport system (ETS) increases with exercise.

The clinical relevance of mitochondrial oxidative capacity is underscored by our findings of associations of reduced muscle mitochondrial oxidative capacity with worse physical performance on the 6-minute walk test. Despite differences in associations of CKD with ATPmax in the hand and leg muscles, ATPmax in both muscle groups was associated with similar differences 6-minute walk distance among patients with CKD. These findings suggest that ${ }^{31} \mathrm{P}$ MRS measurements provide a window into skeletal muscle health and patient-centered outcomes beyond muscle strength that could serve as important indicators of muscle function and physical performance in CKD.

Strengths of the current investigation include direct measurements of skeletal muscle mitochondrial energetics in humans, evaluation of a nondialysis CKD population, selection of control individuals with a similar prevalence of diabetes, and demonstration of associations with physical performance measures. The ${ }^{31} \mathrm{P}$ MRS method used in this investigation has also been applied to other populations in studies validating the association of muscle mitochondrial energetics with patient-centered outcomes and metabolic phenotypes (34-36). One limitation of our study is the cross-sectional design, limiting discovery of potential mechanisms to explain the observed reductions in mitochondrial capacity. Future longitudinal studies that combine in situ and in vivo muscle mitochondrial measures with follow up of physical functioning would be an important next step to address underlying mechanisms linking kidney function, mitochondrial energetics, and functional decline. A significant number of participants were unable to undergo leg muscle ATPmax testing due to technical limitations of the procedure. This may have resulted in a select group of participants who were able to undergo leg muscle testing due to body habitus limitations, reducing the generalizability of our findings to the broader CKD population. It also remains possible that study participants with $\mathrm{CKD}$ and control individuals may have differed by characteristics unrelated to kidney disease that themselves may be related to skeletal muscle mitochondrial energetics. Finally, another limitation of our study was the use of estimated glomerular filtration as an 


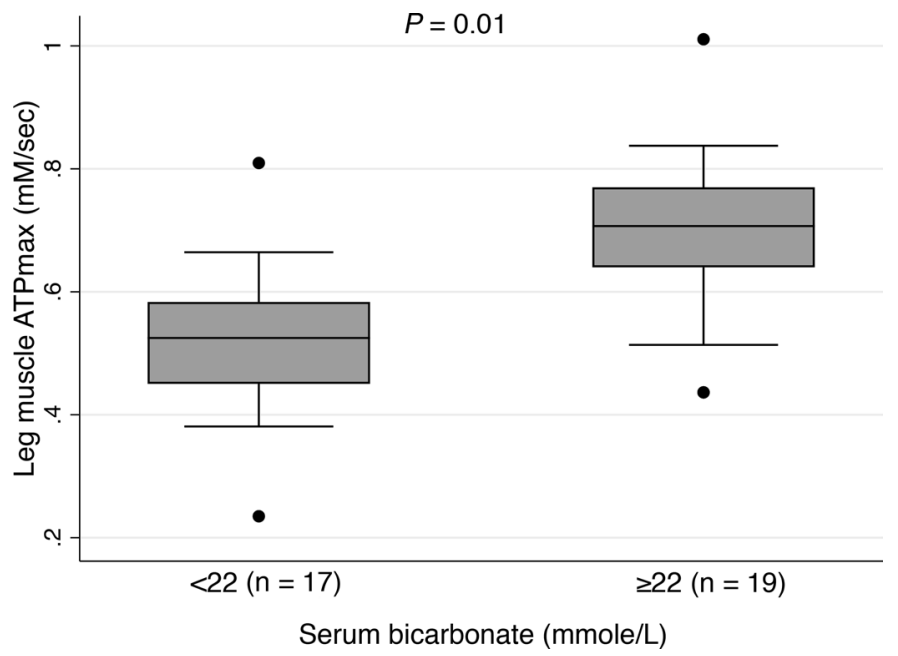

Figure 4. Association of serum bicarbonate level with leg muscle ATPmax among those with eGFR $<\mathbf{6 0}$. Serum bicarbonate levels of less than $22 \mathrm{mmol} / \mathrm{L}$ and more than or equal to $22 \mathrm{mmol} / \mathrm{L}$ from the Kidney Disease: Improving Global Outcomes recommended clinical cut point for bicarbonate repletion therapy in kidney disease. $P$ values were adjusted for age, sex, and eGFR using multivariable linear regression.

index of kidney function. This does not completely capture the secretory function of the kidney, which represents the major mechanism for secretion of protein-bound uremic toxins, whose generation can be influenced by differences in diet and gut microbiota (37).

In conclusion, we demonstrated impaired leg muscle mitochondrial oxidative capacity in patients with CKD using ${ }^{31} \mathrm{P}$ MRS. Impairment in muscle mitochondrial oxidative capacity may underlie poor physical performance, leading to functional decline and frailty in CKD. Further investigation is necessary to help guide therapeutic development and better inform clinical trials aimed at improving skeletal muscle function, physical functioning, and other patient-centered health-related outcomes in CKD.

\section{Methods}

Study population. The Chronic Kidney Disease Muscle Mitochondrial ENergetics and Dysfunction (CKDMEND) study recruited patients with moderate-to-advanced CKD from nephrology clinics and control individuals without known kidney disease from primary care and endocrinology clinics within the University of Washington healthcare system. For the purposes of enrollment, we defined CKD at entry by an eGFR $<60 \mathrm{ml} / \mathrm{min}$ per $1.73 \mathrm{~m}^{2}$ based on at least 2 clinical laboratory evaluations, using the $2009 \mathrm{CKD}$ EPI equation. We defined non-CKD by an eGFR $\geq 60 \mathrm{ml} / \mathrm{min}$ per $1.73 \mathrm{~m}^{2}$ without evidence of albuminuria. We frequency matched $\mathrm{CKD}$ and control participants based on demographics and diabetes status to increase the degree of similarity in these characteristics between the groups. We identified controls in age groups of $40-59$ years of age and 60-80 years of age seeking to match $50 \%$ females and $50 \%$ diabetic patients within in each age group. We further used statistical adjustment to provide finer control of potential confounding due to residual imbalance between study groups.

Exclusion criteria were age of $<18$ years, history of solid-organ transplantation, inability to ambulate at least 100 steps, being treated with maintenance dialysis or being expected to start dialysis within 6 months, uncontrolled hypertension (systolic $\mathrm{BP}>170 \mathrm{mmHg}$ ), history of deep venous thrombosis or pulmonary embolism, use of dual antiplatelet therapy, history of HIV or viral hepatitis infection, uncontrolled thyroid disease (elevated or depressed TSH), weight $>300 \mathrm{lbs}$, oxygen-dependent chronic obstructive pulmonary disease, active malignancy, vascular stent placed within 6 months, and any MRI incompatible devices or implants. We also excluded individuals who were treated with medications that have known effects on muscle metabolism, physical function, or mitochondrial metabolism, including antivirals, muscle relaxants, antipsychotics, oral steroids, calcineurin inhibitors, antiepileptics, coenzyme Q-10, nicotinamide riboside, or high-dose antioxidants. Finally, we limited MRI studies of the leg to persons who were at least 5 foot 2 inches in height due to the small-bore size of the MRI.

Measurements of muscle mitochondrial energetics. To measure mitochondrial ATPmax in a consistent volume of muscle tissue, we used the time course of recovery of phosphocreatine after exercise; for this, we used ${ }^{31} \mathrm{P}$ MRS of the tibialis anterior leg muscle and FDI hand muscle $(38,39)$ with a standardized exercise protocol used to assess muscle oxidative capacity and ATP kinetics in multiple populations $(34,36,40)$. Briefly, a short exercise test involving repeated dorsiflexion of the right foot or index finger of the right hand 


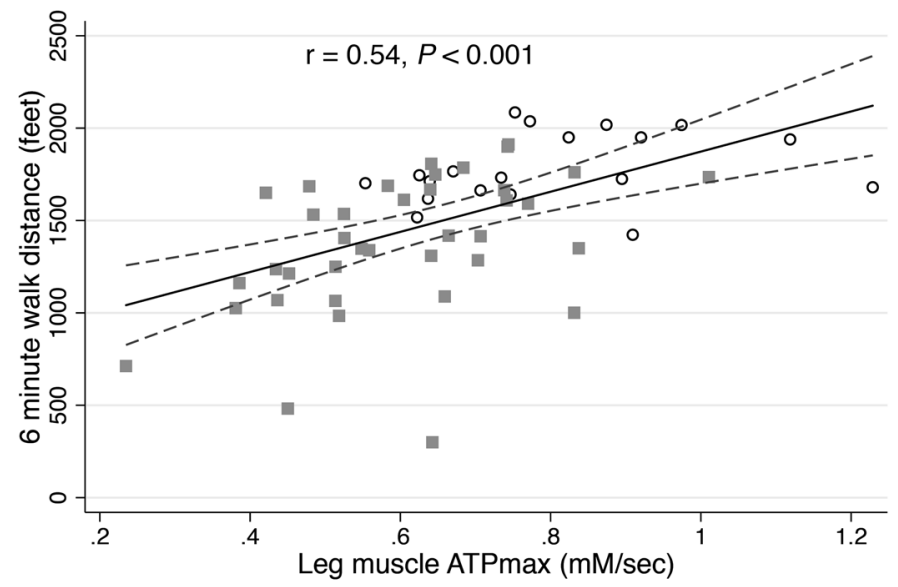

\begin{tabular}{|l|c|c|}
\hline o Control & CKD & P-value \\
\hline & $r$ & 0.005 \\
\hline CKD & 0.44 & 0.28 \\
\hline Control & 0.26 & \\
\hline
\end{tabular}

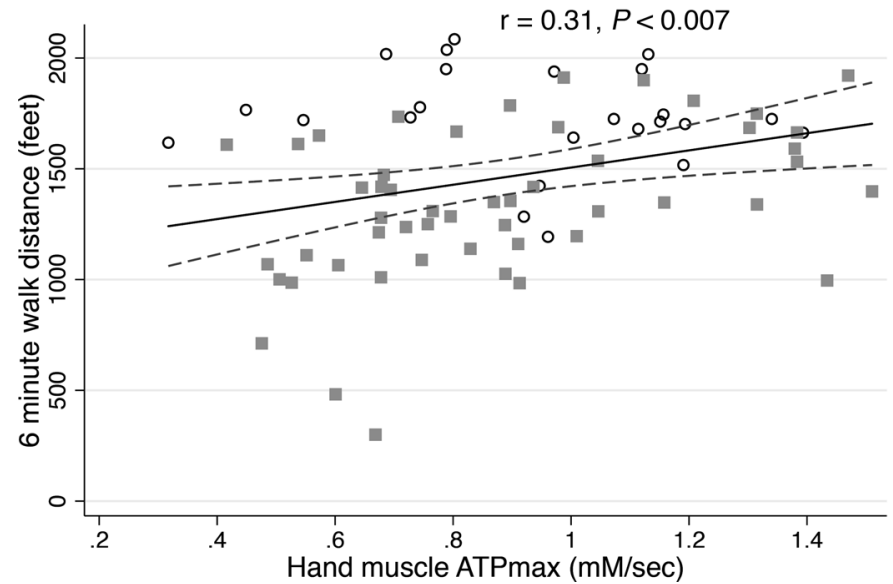

\begin{tabular}{|l|c|c|}
\hline o Control & CKD & P-value \\
\hline & $r$ & 0.002 \\
\hline CKD & 0.43 & 0.58 \\
\hline Control & -0.12 & $\mathrm{Cl}$ \\
\hline
\end{tabular}

Figure 5. Leg and hand muscle mitochondrial capacity are associated with 6-minute walk distance. Association of leg and hand muscle ATPmax with physical performance. $P$ for interaction by CKD status $=0.04$ for leg muscle and $P$ for interaction $=0.02$ for hand muscle ATPmax using likelihood ratio test.

was performed for a period of 20 to 30 seconds to reduce phosphocreatine by approximately $50 \%$, while maintaining muscle $\mathrm{pH}>6.8$. The phosphocreatine recovery was measured over 6 minutes to determine a time constant of recovery $\left(\mathrm{t}_{\mathrm{PCr}}\right)$ to yield ATPmax (ATPmax $=\left[24.5 / \mathrm{t}_{\mathrm{PCr}}\right]$ for the FDI or $\left[26.5 / \mathrm{t}_{\mathrm{PCr}}\right]$ for the tibialis anterior), where PCR denotes phosphocreatine. The ATPmax is the standard for characterizing mitochondrial ATP capacity in vivo $(39,41,42)$ in a consistent muscle volume of interest and is directly related both to mitochondrial markers of oxidative phosphorylation in muscle tissue (43-45) and in vivo (46). We also measured FDI hand resting muscle oxygen uptake and net ATPflux in resting muscle during ischemia. Briefly, resting ATPflux was determined by MRS, with the dynamics and concentration of the high-energy phosphate carrier phosphocreatine during anoxia in the muscle $(30,47)$. All MRS was performed on a Bruker 4.7-T magnet with Biospin console. The within-patient reproducibility of ${ }^{31} \mathrm{P}$ MRS leg ATPmax, our principal measure of muscle energetics, was less than $10 \%$, in agreement with that of other investigators ( $5 \%-8 \%$ within-person day-to-day coefficient of variation) $(48,49)$, while, for the hand, the coefficient of variation was $13 \%$. The within-patient coefficient of variation for ATPflux was $14 \%$.

Measurement of resting oxygen uptake. The same FDI hand muscle was used in optical spectroscopy and MRS in a subset of participants during a separate is ischemia experiment performed on the same day as the MRS. Participants underwent a 15-minute ischemic procedure where a blood pressure cuff was inflated to $50 \mathrm{mmHg}$ above the participants systolic blood pressure. This muscle has the advantage of having very low adipose tissue thickness ( $2 \mathrm{~mm}$ ). Nonetheless a reference using $2 \%$ intralipid was used to estimate scattering due to adipose tissue penetration. The spectroscopic depth of penetration is approximately $5 \mathrm{~mm}$, and the volume of muscle sampled approximates that sampled by the surface coil used for ${ }^{31} \mathrm{P}$ MRS.

Oxygen $\left(\mathrm{O}_{2}\right)$ uptake by muscle was measured using the deoxygenation rate of myoglobin $(\mathrm{Mb})$ to $\mathrm{Hb}$ with optical spectroscopy. This was assessed as the drop in $\mathrm{O}_{2}$ content of each carrier $(\Delta$ saturation $\times \mathrm{Mb}$ or $\mathrm{Hb}$ ) 
Table 2. Association of leg muscle ATPmax with 6-minute walk distance performance in feet

\begin{tabular}{|c|c|c|}
\hline Leg muscle & $\begin{array}{c}\text { Unadjusted }(n=57) \\
\beta(95 \% \mathrm{CI})\end{array}$ & $\begin{array}{l}\text { Model } 1(n=52) \\
\quad \beta(95 \% \mathrm{Cl})\end{array}$ \\
\hline TA muscle ATPmax (per 1 mM/s) & $1087(642,1530)$ & $756(222,1290)$ \\
\hline Age (per 1 yr) & & $-9.3(-18.8,0.2)$ \\
\hline Female & & $-11.6(-285,308)$ \\
\hline $\mathrm{BMI}$ & & $-17.9(-43.6,7.8)$ \\
\hline Diabetes & & $52.3(-183.4,288)$ \\
\hline Muscle size in $\mathrm{mm}^{2}$ & & $0.1(-0.6,0.8)$ \\
\hline Muscle strength in newtons & & $2.6(-0.4,5.7)$ \\
\hline
\end{tabular}

during ischemia. The desaturation rate was determined during the first 2 minutes of ischemia, when $\mathrm{Mb}-\mathrm{O}_{2}$ is $>50 \%$ saturated (partial pressure of oxygen $\left[\mathrm{PO}_{2}\right]>3$ torr), and mitochondrial respiration is independent of cellular $\mathrm{PO}_{2}(50,51)$. Unlike in NIRS, the analyses of the optical spectra used a partial least squares approach based on the second derivative of the spectra, allowing separation of the signals from oxygenated and deoxygenated $\mathrm{Mb}$ and from oxygenated and deoxygenated $\mathrm{Hb}$, yielding a more specific surrogate for muscle oxygen consumption (52). The within-patient coefficient of variation in oxygen uptake was $20 \%$.

Measurement of hand muscle resting ATPflux. The resting ATPflux (ATPase rate) was determined from the phosphocreatine breakdown in the hand muscle during the last 3 minutes of a separate 15 -minute ischemic protocol performed before the oxygen uptake experiments with the same blood pressure cuff inflation pressure as in the optical spectroscopy experiments. During the last 3 minutes of ischemia the slope of the change in phosphocreatine represent the flux that must be met by mitochondrial oxidative phosphorylation under aerobic conditions to achieve ATP balance as previously described (47). This breakdown is a direct measure of ATPflux because phosphocreatine breakdown acts as a buffer to maintain ATP levels in the face of energy demand via the creatine kinase reaction.

Measurements of muscle size and strength. We measured leg and hand muscle size cross-sectional area (CSA) from MR images acquired as axial plane $\mathrm{T}_{1}$-weighted, 2D gradient-echo images; these were collected with the following parameters: 500-ms repetition time, 2.5-ms echo time, 3-mm slice thickness, 1-mm interslice interval, $192 \times 192$ matrix, and number of excitations $=2$. Five slices of each right limb were analyzed with Image J (version $1.50 \mathrm{e}, \mathrm{NIH}$ ) using manual planimetry (53) to determine the total muscle CSA. Measurements by 2 independent investigators agreed to within $2.5 \%$ on average. The mean of the maximal 3 muscle CSA measures was used in the analysis. Tibialis anterior and FDI muscle strength and contractile properties were determined on the right leg or hand using a custom-built isometric exercise apparatus, as previously described (54). The tibialis anterior muscle is important in ankle dorsiflexion and represents a simple system connecting muscle mechanics and energetics to foot movement (54), which serves a critical role in walking mobility. The subject performed a maximal voluntary contraction in ankle dorsiflexion exercise for approximately 5 seconds by pulling on a strap that secured the foot to a force transducer platform. Three successive bouts were separated by 5 seconds each.

Measurements of physical performance. We performed a 6-minute walk test within 28 days of muscle testing. Participant were instructed to walk as fast as possible on a flat surface in a well-lit marked indoor corridor free of pedestrian traffic, as previously described (55). Participants were told to cover as much distance as possible over 6 minutes. Research coordinators followed a standardized script for performing testing to avoid differences in the level of encouragement.

Measurements of other study data. Study coordinators measured seated blood pressures 5 minutes apart using an automated sphygmomanometer and recorded the average of the last 2 readings. Coordinators collected blood and 12-hour timed urine samples on the same day as the physical performance evaluation. Samples were centrifuged for 20 minutes at $1728 \mathrm{~g}$, transferred to cryovials, and stored at $-80^{\circ} \mathrm{C}$. General chemistries were measured from frozen sera on a Beckman Coulter DXC autoanalyzer. Serum creatinine concentrations were measured with values traceable to isotope dilution mass spectrometry standards. Serum cystatin $C$ concentrations were measured using a Gentian particle-enhanced turbidometric assay standardized to international reference material ERM-DA471. Study measurements of eGFR were estimated using serum creatinine and cystatin C, based on 
Table 3. Association of hand muscle ATPmax with 6-minute walk distance performance in feet

\begin{tabular}{|c|c|c|}
\hline Hand muscle & $\begin{array}{c}\text { Unadjusted }(n=75) \\
\beta(95 \% \mathrm{CI})\end{array}$ & $\begin{array}{l}\text { Model } 1(n=70) \\
\quad \beta(95 \% \mathrm{Cl})\end{array}$ \\
\hline FDI ATPmax (per 1mM/s) & $387(111,663)$ & $399(145,654)$ \\
\hline Age (per 1 yr) & & $-7.8(-13.5,-2.0)$ \\
\hline Female & & $-164(-322,-5.8)$ \\
\hline $\mathrm{BMI}$ & & $-19.4(-30.2,-8.5)$ \\
\hline Diabetes & & $-77(-241,87)$ \\
\hline Muscle size in $\mathrm{mm}^{2}$ & & $1.3(0.1,2.5)$ \\
\hline Muscle strength in newtons & & $-2.1(-8.2,4.1)$ \\
\hline
\end{tabular}

the 2012 combined CKD-EPI equation (56). We measured urinary albumin concentration by immunoturbidimetry and urinary creatinine concentration by the modified Jaffe method.

Statistics. We performed complete case analysis of participants with complete endpoint data (Supplemental Figure 1). Less than $10 \%$ of those who had completed ${ }^{31} \mathrm{P}$ MRS measurements of ATPmax had missing muscle strength due to the inability to fit into the muscle testing apparatus or poor quality surface coil imaging of the tibialis anterior muscle. We tested differences in key demographic characteristics between participants with $\mathrm{CKD}$ and controls for different endpoint subgroups using the 2-sample 2-tailed $t$ test. We used scatterplots, simple linear regression, and Pearson's correlation to describe associations of kidney function with MRS measures of interest. We used linear regression to estimate associations between kidney function and ATPmax, adjusting for confounders, including age, sex, BMI, and diabetes. A $P$ value of less than 0.05 was considered significant. We chose not to adjust for muscle size or strength in our main models but rather to do so in sensitivity analyses because ${ }^{31} \mathrm{P}$ MRS was performed over a consistent muscle volume of interest and because prevailing evidence suggests that kidney disease may contribute to muscle atrophy. We also used linear regression to estimate associations of ATPmax with 6-minute walking distance. We tested for effect modification using the likelihood ratio test. Consistent with previous studies (34), we used whole muscle oxidative capacity (defined as a product of ATPmax with muscle cross-sectional area) as the main exposure or outcome in sensitivity analysis. For our sensitivity analysis, which tested the association of eGFR with whole leg muscle oxidative capacity, we log transformed ATPmax ${ }^{*}$ CSA in order to determine the association of GFR with the percentage of change in whole muscle oxidative capacity, defined as $\left(\mathrm{e}^{\beta}-1\right) \times 100$, where $\mathrm{e}$ is the exponential function of the regression coefficient $(\beta)$ for the association of eGFR with log-transformed ATPmax ${ }^{*}$ CSA obtained from the linear regression models. Values for high sensitivity C-reactive protein were skewed and therefore log transformed to normalize their distribution. Statistical analyses were performed using STATA version 15.

Study approval. The CKD-MEND study protocol was approved by the University of Washington Human Subjects Division. All participants provided informed consent prior to participation in the CKDMEND study.

\section{Author contributions}

$\mathrm{BR}, \mathrm{BK}, \mathrm{KC}, \mathrm{IHDB}$, and JH designed and implemented the study. SL, ASA, ES, and KC carried out the experiments. BR, BK, and JG analyzed the data. BR and BK made the figures. BR, JG, BK, IHDB, JH, $\mathrm{KC}, \mathrm{TJ}, \mathrm{CG}$, and LS drafted and revised the paper.

\section{Acknowledgments}

The investigators would like to thank all the participants and study coordinators who dedicated their time to the CKD-MEND study. We express our gratitude for the work of Laura Curtin, Connor Henry, Nicole Robinson, and Ernest Ayers for their efforts in recruitment and management of the project. We are grateful for David J. Marcinek's review and editing of the manuscript. Funding for this investigation was obtained from the National Institute of Diabetes and Digestive and Kidney Diseases: R01 DK101509 (to BK), K23 DK0099442 (to BR), R03 DK114502 (to BR), K23 DK100533 (to JG), Dialysis Clinic Inc. (C-4112, to BR), and R01 DK099199 (to IHDB). This project was further supported by a grant from Puget Sound Veterans Affairs Health Care System and an unrestricted grant from the Northwest Kidney Centers. 
Address correspondence to: Baback Roshanravan, UCD, 451 Health Sciences Drive, Suite 5321, Davis, California 95616, USA. Phone: 530.754.0893; Email: broshanr@ucdavis.edu.

1. Ramer SJ, et al. Health outcome priorities of older adults with advanced CKD and concordance with their nephrology providers' perceptions. J Am Soc Nephrol. 2018;29(12):2870-2878.

2. Tamaki M, Miyashita K, Wakino S, Mitsuishi M, Hayashi K, Itoh H. Chronic kidney disease reduces muscle mitochondria and exercise endurance and its exacerbation by dietary protein through inactivation of pyruvate dehydrogenase. Kidney Int. 2014;85(6):1330-1339.

3. Roshanravan B, et al. Chronic kidney disease attenuates the plasma metabolome response to insulin. JCI Insight. 2018;3(16):122219.

4. Conjard A, Ferrier B, Martin M, Caillette A, Carrier H, Baverel G. Effects of chronic renal failure on enzymes of energy metabolism in individual human muscle fibers. J Am Soc Nephrol. 1995;6(1):68-74.

5. Afshinnia F, et al. Impaired $\beta$-oxidation and altered complex lipid fatty acid partitioning with advancing CKD. J Am Soc Nephrol. 2018;29(1):295-306

6. Roshanravan B, et al. CKD and muscle mitochondrial energetics. Am J Kidney Dis. 2016;68(4):658-659.

7. Manfredini F, et al. The role of deconditioning in the end-stage renal disease myopathy: physical exercise improves altered resting muscle oxygen consumption. Am J Nephrol. 2015;41(4-5):329-336.

8. Kemp GJ, et al. Abnormal mitochondrial function and muscle wasting, but normal contractile efficiency, in haemodialysed patients studied non-invasively in vivo. Nephrol Dial Transplant. 2004;19(6):1520-1527.

9. Lemoine $\mathrm{S}$, et al. Intracellular phosphate dynamics in muscle measured by magnetic resonance spectroscopy during hemodialysis. J Am Soc Nephrol. 2016;27(7):2062-2068.

10. Marrades RM, et al. Cellular bioenergetics after erythropoietin therapy in chronic renal failure. J Clin Invest. 1996;97(9):2101-2110.

11. Thompson RT, Muirhead N, Marsh GD, Gravelle D, Potwarka JJ, Driedger AA. Effect of anaemia correction on skeletal muscle metabolism in patients with end-stage renal disease: 31P magnetic resonance spectroscopy assessment. Nephron. 1996;73(3):436-441.

12. Sun CY, Cheng ML, Pan HC, Lee JH, Lee CC. Protein-bound uremic toxins impaired mitochondrial dynamics and functions. Oncotarget. 2017;8(44):77722-77733.

13. Thome $\mathrm{T}$, et al. Uremic metabolites impair skeletal muscle mitochondrial energetics through disruption of the electron transport system and matrix dehydrogenase activity. Am J Physiol, Cell Physiol. 2019;317(4):C701-C713.

14. Koppe L, et al. p-Cresyl sulfate promotes insulin resistance associated with CKD. J Am Soc Nephrol. 2013;24(1):88-99.

15. Mutsaers HA, et al. Uremic toxins inhibit renal metabolic capacity through interference with glucuronidation and mitochondrial respiration. Biochim Biophys Acta. 2013;1832(1):142-150.

16. May RC, Kelly RA, Mitch WE. Metabolic acidosis stimulates protein degradation in rat muscle by a glucocorticoid-dependent mechanism. J Clin Invest. 1986;77(2):614-621.

17. Bailey JL, Wang X, England BK, Price SR, Ding X, Mitch WE. The acidosis of chronic renal failure activates muscle proteolysis in rats by augmenting transcription of genes encoding proteins of the ATP-dependent ubiquitin-proteasome pathway. J Clin Invest. 1996;97(6):1447-1453.

18. [No authors listed]. Chapter 3: Management of progression and complications of CKD. KDIGO 2012 Clinical Practice Guidelines for the Evaluation and Management of Chronic Kidney Disease. Kidney Int Suppl. 2013;3(1):73-90.

19. Wesson DE, et al. Long-term safety and efficacy of veverimer in patients with metabolic acidosis in chronic kidney disease: a multicentre, randomised, blinded, placebo-controlled, 40-week extension. Lancet. 2019;394(10196):396-406.

20. Dubey AK, Sahoo J, Vairappan B, Haridasan S, Parameswaran S, Priyamvada PS. Correction of metabolic acidosis improves muscle mass and renal function in chronic kidney disease stages 3 and 4: a randomized controlled trial. Nephrol Dial Transplant. 2020;35(1):121-129.

21. Franch HA, Raissi S, Wang X, Zheng B, Bailey JL, Price SR. Acidosis impairs insulin receptor substrate-1-associated phosphoinositide 3-kinase signaling in muscle cells: consequences on proteolysis. Am J Physiol Renal Physiol. 2004;287(4):F700-F706.

22. Bailey JL, Zheng B, Hu Z, Price SR, Mitch WE. Chronic kidney disease causes defects in signaling through the insulin receptor substrate/phosphatidylinositol 3-kinase/Akt pathway: implications for muscle atrophy. J Am Soc Nephrol. 2006;17(5):1388-1394.

23. Das S, et al. ATP citrate lyase improves mitochondrial function in skeletal muscle. Cell Metab. 2015;21(6):868-876.

24. Echtay KS, et al. Superoxide activates mitochondrial uncoupling proteins. Nature. 2002;415(6867):96-99.

25. Siegel MP, et al. Mitochondrial-targeted peptide rapidly improves mitochondrial energetics and skeletal muscle performance in aged mice. Aging Cell. 2013;12(5):763-771.

26. Talbert EE, Smuder AJ, Min K, Kwon OS, Szeto HH, Powers SK. Immobilization-induced activation of key proteolytic systems in skeletal muscles is prevented by a mitochondria-targeted antioxidant. J Appl Physiol. 2013;115(4):529-538.

27. Fazakerley DJ, et al. Mitochondrial $\mathrm{CoQ}$ deficiency is a common driver of mitochondrial oxidants and insulin resistance. Elife. 2018;7:e32111.

28. Zheng L, et al. Fumarate induces redox-dependent senescence by modifying glutathione metabolism. Nat Commun. 2015;6:6001.

29. Moore GE, Bertocci LA, Painter PL. 31P-magnetic resonance spectroscopy assessment of subnormal oxidative metabolism in skeletal muscle of renal failure patients. J Clin Invest. 1993;91(2):420-424.

30. Amara CE, Shankland EG, Jubrias SA, Marcinek DJ, Kushmerick MJ, Conley KE. Mild mitochondrial uncoupling impacts cellular aging in human muscles in vivo. Proc Natl Acad Sci USA. 2007;104(3):1057-1062.

31. Schiaffino S, Reggiani C. Fiber types in mammalian skeletal muscles. Physiol Rev. 2011;91(4):1447-1531.

32. Brand MD, Nicholls DG. Assessing mitochondrial dysfunction in cells. Biochem J. 2011;435(2):297-312.

33. Brand MD, Esteves TC. Physiological functions of the mitochondrial uncoupling proteins UCP2 and UCP3. Cell Metab. 
2005;2(2):85-93.

34. Santanasto AJ, et al. Skeletal muscle mitochondrial function and fatigability in older adults. J Gerontol A Biol Sci Med Sci. 2015;70(11):1379-1385.

35. Coen PM, et al. Skeletal muscle mitochondrial energetics are associated with maximal aerobic capacity and walking speed in older adults. J Gerontol A Biol Sci Med Sci. 2013;68(4):447-455.

36. Fabbri E, et al. Insulin resistance is associated with reduced mitochondrial oxidative capacity measured by 31P-magnetic resonance spectroscopy in participants without diabetes from the baltimore longitudinal study of aging. Diabetes. 2017;66(1):170-176.

37. Ramezani A, Raj DS. The gut microbiome, kidney disease, and targeted interventions. J Am Soc Nephrol. 2014;25(4):657-670.

38. Amara CE, Marcinek DJ, Shankland EG, Schenkman KA, Arakaki LS, Conley KE. Mitochondrial function in vivo: spectroscopy provides window on cellular energetics. Methods. 2008;46(4):312-318.

39. Conley KE, Jubrias SA, Esselman PC. Oxidative capacity and ageing in human muscle. J Physiol (Lond). 2000;526 Pt 1:203-210.

40. Braganza A, et al. Platelet bioenergetics correlate with muscle energetics and are altered in older adults. JCI Insight. 2019;5:128248.

41. Jubrias SA, Esselman PC, Price LB, Cress ME, Conley KE. Large energetic adaptations of elderly muscle to resistance and endurance training. J Appl Physiol. 2001;90(5):1663-1670.

42. Broskey NT, et al. Skeletal muscle mitochondria in the elderly: effects of physical fitness and exercise training. J Clin Endocrinol Metab. 2014;99(5):1852-1861.

43. McCully KK, Fielding RA, Evans WJ, Leigh JS, Posner JD. Relationships between in vivo and in vitro measurements of metabolism in young and old human calf muscles. J Appl Physiol. 1993;75(2):813-819.

44. Conley KE, et al. Higher mitochondrial respiration and uncoupling with reduced electron transport chain content in vivo in muscle of sedentary versus active subjects. J Clin Endocrinol Metab. 2013;98(1):129-136.

45. Gonzalez-Freire M, et al. Skeletal muscle ex vivo mitochondrial respiration parallels decline in vivo oxidative capacity, cardiorespiratory fitness, and muscle strength: The Baltimore Longitudinal Study of Aging. Aging Cell. 2018;17(2):e12725.

46. Ryan TE, Southern WM, Reynolds MA, McCully KK. A cross-validation of near-infrared spectroscopy measurements of skeletal muscle oxidative capacity with phosphorus magnetic resonance spectroscopy. J Appl Physiol. 2013;115(12):1757-1766.

47. Amara CE, Marcinek DJ, Shankland EG, Schenkman KA, Arakaki LS, Conley KE. Mitochondrial function in vivo: spectroscopy provides window on cellular energetics. Methods. 2008;46(4):312-318.

48. Sleigh A, et al. Simple and effective exercise design for assessing in vivo mitochondrial function in clinical applications using (31)P magnetic resonance spectroscopy. Sci Rep. 2016;6:19057.

49. Larson-Meyer DE, Newcomer BR, Hunter GR, Hetherington HP, Weinsier RL. 31P MRS measurement of mitochondrial function in skeletal muscle: reliability, force-level sensitivity and relation to whole body maximal oxygen uptake. NMR Biomed. 2000;13(1):14-27.

50. Marcinek DJ. Mitochondrial dysfunction measured in vivo. Acta Physiol Scand. 2004;182(4):343-352.

51. Marcinek DJ, Ciesielski WA, Conley KE, Schenkman KA. Oxygen regulation and limitation to cellular respiration in mouse skeletal muscle in vivo. Am J Physiol Heart Circ Physiol. 2003;285(5):H1900-H1908.

52. Arakaki LS, Ciesielski WA, Thackray BD, Feigl EO, Schenkman KA. Simultaneous optical spectroscopic measurement of hemoglobin and myoglobin saturations and cytochrome aa3 oxidation in vivo. Appl Spectrosc. 2010;64(9):973-979.

53. Trappe TA, Lindquist DM, Carrithers JA. Muscle-specific atrophy of the quadriceps femoris with aging. J Appl Physiol. 2001;90(6):2070-2074.

54. Jubrias SA, Crowther GJ, Shankland EG, Gronka RK, Conley KE. Acidosis inhibits oxidative phosphorylation in contracting human skeletal muscle in vivo. J Physiol (Lond). 2003;553(Pt 2):589-599.

55. Roshanravan B, et al. Association between physical performance and all-cause mortality in CKD. J Am Soc Nephrol. 2013;24(5):822-830.

56. Inker LA, et al. Estimating glomerular filtration rate from serum creatinine and cystatin C. N Engl J Med. 2012;367(1):20-29. 